\title{
Global vernetzt, lokal engagiert: Trinationales Begegnungsprojekt mit (angehenden) Sozialarbeiter/-innen in Jordanien
}

Lautes Stimmen- und Sprachengewirr: Eine angeregte Diskussion, erst auf Englisch, dann mit arabischer Übersetzungspause, Zwischenüberlegungen auf Deutsch. Am Ende steht nicht unbedingt ein Konsens - aber der Gewinn neuer Perspektiven, durch den sich Diskussionsräume für weiteren Austausch eröffnen. Und genau das war die Idee der Organisatorinnen des trinationalen Begegnungsprojekts im Sommer 2019 in Jordanien: Den (angehenden) Sozialarbeiter/-innen Raum für professionellen Austausch bieten, persönliches Kennenlernen ermöglichen und dabei über die Chancen und Möglichkeiten der Agenda 2030 für die Soziale Arbeit weltweit, aber insbesondere auf der lokalen (Handlungs-)Ebene nachdenken. Diskussionen, Praxisbesuche und Ausflüge vernetzten die Teilnehmenden und boten Inspiration für weitere Intervention in den jeweiligen Herkunftsregionen in Jordanien, den Palästinensischen Autonomiegebieten und in Deutschland. Neben der Auseinandersetzung mit den Nachhaltigkeitszielen der Vereinten Nationen stand auch der Austausch über Gemeinsamkeiten und Unterschiede der Sozialen Arbeit als Disziplin und Profession in Deutschland und im arabischen Raum im Fokus. Das Programm beinhaltete neben Praxisbesuchen auch Vorlesungen von Dozierenden aus Bethlehem, Amman und Köln, die den Teilnehmenden einen Einblick in ihre Forschungs- und Lehrtätigkeit gewährten. Dabei setzten sich die Professor/-innen und Studierenden in Form eines Workshopcharakters mit unterschiedlichen Themen auseinander: Es ging um die Notwendigkeit von professionellen Haltungen in der Sozialen Arbeit unter besonderer Berücksichtigung von Einstellungen wie Openess, Respect, Empathy, Authenticity, Ambiguity. Auch die Relevanz von Fachkräfteaustauschen wurde beleuchtet; ebenso die Bedeutung von historischen Zusammenhängen für aktuelle Handlungspraktiken sowie Flucht und Migration als globales Thema. Bei jedem Workshop gab es eine ausgewogene Mischung aus theoretischem Input und Beiträgen aus praktischen Erfahrungen aus verschiedenen Ländern.

Die Durchführung des Projekts in Jordanien ermöglichte es den Teilnehmenden, neben theoretischen Inputs auch einen Eindruck der sozialarbeiterischen Praxis vor Ort zu erhalten. Im Verlauf des fünftägigen Projekts wurden verschiedene Einrichtungen der Sozialen Arbeit in Jordanien besucht, deren Konzepte lokal geprägt, jedoch global anwendbar sind. Es sind in diesem Sinne „glokale Projekte“, die ökologische und soziale Ziele in den Vordergrund stellen. Darunter war zum Beispiel das „Mercy Restaurant“ der Caritas Jordanien im Zentrum Ammans, das täglich 300-500 kostenlose Mahlzeiten an
Menschen mit geringen sozioökonomischen Ressourcen verteilt. Außerdem stand der Besuch des sozialökologischen Umweltbildungsprojekts „Al Numeira“ am Toten Meer auf dem Programm, bei dem sich die Teilnehmenden über die Möglichkeiten der Einbindung der lokalen Community in Bildungsund Umweltprojekte informieren konnten. Besonders eindrücklich und inspirierend erwies sich ein Besuch der „Shams Community“, die im Zentrum Ammans wöchentliche Dialogtreffen in Form von Leitfragediskussionen mit anschließendem gemeinsamem Essen anbietet. Die Teilnehmenden diskutierten hier in unterschiedlichen Gruppen und Konstellationen mit Jordanier/innen und Gästen aus aller Welt über Sustainable Development aus sozialer, wirtschaftlicher, ökologischer und entwicklungspolitischer Perspektive. Diese Veranstaltung war für alle Teilnehmenden des Workshops eine inspirierende Erfahrung: Im Anschluss an den Abend wurden die Kontaktadressen mit den Organisator/-innen vor Ort ausgetauscht und es entstanden Pläne zur Implementierung des Gesprächskonzepts in die eigene sozialarbeiterische Praxis.

Die Teilnehmenden des Begegnungsprojekts einte vor allem eines: die Disziplin und vor allem die Profession Soziale Arbeit. In Diskussionsplenen und Kleingruppenarbeit kristallisierte sich zunehmend heraus, dass Themen wie eine gelungene Balance von Nähe und Distanz, der Rückgriff auf adäquate Theorien der Sozialen Arbeit und die Selbstreflexion und Supervision im Praxisalltag unabhängig von kulturellen und nationalen Identitäten eine Rolle spielen. Die Beschäftigung mit den 17 Nachhaltigkeitszielen der Vereinten Nationen bestärkte die Teilnehmenden darin, ihre eigene professionelle Rolle bezüglich Empowerment, sozioökonomischer Entwicklung, Menschenrechten und der Umwelt neu zu definieren. Offensichtlich konnten die Erwartungen der (angehenden) Sozialarbeiter/-innen insgesamt erfüllt werden - obwohl natürlich zahlreiche Aspekte nicht in der gewünschten Ausführlichkeit diskutiert werden konnten. Deshalb stand am Ende des Begegnungsprojekts die Frage nach einer Neuauflage im kommenden Jahr zur Debatte. Ein konkreter Output des Projekts ist außerdem die Realisierung eines thematisch am Begegnungsprojekt orientierten Panels einiger Teilnehmender auf der diesjährigen Konferenz der International Federation of Social Workers, die aufgrund der Covid-19-Pandemie nicht wie geplant in Calgary/Kanada, sondern online stattfand.

Julia Frericks \& Milena Feldmann doi.org/10.31244/zep.2020.04.08 